\title{
ENSENIAS: HERRAMIENTA TECNOLÓGICA PARA APRENDER, ENSEÑAR, MEJORAR Y USAR LA LENGUA DE SIGNOS PANAMEÑA
}

\author{
EnSenias: Technological tool to learn, teach, improve and use Panamanian Sign \\ LANGUAGE
}

ENSENIAS: UN OUTIL TECHNOLOGIQUE POUR APPRENDRE, ENSEIGNER, AMÉLIORER ET UTILISER LA LANGUE DES SIGNES PANAMÉENNE

\author{
Antonio Rodríguez-Fuentes \\ Ph. D. en Pedagogía, Universidad de \\ Granada, España. \\ Profesor titular, Facultad de Ciencias \\ de la Educación, Universidad de \\ Granada, España. \\ arfuente@ugr.es \\ http://orcid. \\ org/0000-0002-8036-9902

\section{Lineth Alaín} \\ Ph. D. en Ciencias de la Educación \\ Catedrática de la Facultad de \\ Ingeniería y Sistemas de la Universidad \\ Tecnológica de Panamá, Universidad \\ Tecnológica de Panamá, Panamá. \\ lineth.alain@utp.ac.pa \\ https://orcid. \\ org/0000-0002-0961-8822
}

\section{Francisco García García}

Ph. D. en Ciencias de la Información

Catedrático de la Facultad de

Comunicación, Universidad

Complutense de Madrid, España.

fghenche@gmail.com

https://orcid.

org/0000-0001-5394-4804

\begin{abstract}
RESUMEN
La comunicación ha sido una constante en el progreso en la sociedad, y la tecnología se ha erigido, desde hace poco, en su vehículo de expansión. La comunidad sorda encuentra limitada su comunicación y requiere engranajes adicionales. Este estudio de diseño no experimental, descriptivo y de enfoque mixto se enfoca en EnSenias, una plataforma on-line para aprender, enseñar, mejorar y usar la lengua de signos panameña, con el fin de explorar su funcionalidad mediante la validación de la misma. El estudio se llevó a cabo en el Instituto Panameño de Habilitación Especial. Para llevarlo a cabo, se recogieron datos de doce grupos focales (57 participantes) y se realizó un análisis mixto. Los resultados del análisis demuestran que EnSenias es necesaria y adecuada para el aprendizaje de esta lengua, su accesibilidad es universal, es útil para aprender y enseñar vocabulario de signos panameños, y también sirve como diccionario y traductor elemental de dicha lengua. Su carácter accesible permite que ciudadanos sordos, hipoacúsicos, oyentes, ciegos, con baja visión, sordociegos y otros puedan conocer la lengua de signos.
\end{abstract}

Palabras clave: tecnología educativa; plataformas educativas; herramientas tecnológicas; lenguaje de señas; lengua de signos panameña; EnSenias.

\section{Abstract}

Communication has always been a requiremente for society advancement, and technology has recently become a vehicle for its expansion. The deaf community has found some limitations in this area, and, therefore, requires additional support. This mixed, non-experimental, descriptive study focuses on EnSenias, an online platform that can be used to learn, teach, improve, and use Panamanian sign language, and has as its main purpose to explore its functionality and validity. This study was carried out at the Panamanian Institute for Special Enabling. To carry it out, data were gathered through twelve focus groups (with 57 participants), and analyzed using mixed analysis techniques. The results of the analysis show that EnSenias is necessary and suitable to learn the sign language, provides universal 
accesibility, is useful to learn and teach sign vocabulary, and serves as an elementary dictionary and translator in sign language. Its affordability allows deaf, hearing-impaired, hearing, blind, short-sighted, and deaf-blind people to learn sign language.

Keywords: educational technology; Panamanian sign language; educational platforms; technological tools; EnSenias.

\section{RÉSUMÉ}

La communication a toujours été le moteur de la société et la technologie est devenue, récemment, l'un de ses rouages. La communauté sourde est limitée dans sa communication et nécessite des dispositifs supplémentaires. On présente «EnSenias», une plateforme en ligne pour apprendre/enseigner/améliorer/ utiliser la langue des signes panaméenne (LSP), étant donné la méconnaissance de cette langue. Elle a été construite au moyen de Recherche-Basée-sur-la-Conception, validée par des analyses mixtes des données recueillies auprès de douze groupes focalisés (57 participants). Cette étude a été menée à l'Instituto Panameño de Habilitación Especial. On montre la nécessité et l'adéquation d' «EnSenias », son accessibilité universelle, son usage pour apprendre et enseigner le vocabulaire des signes, ainsi que son emploi comme dictionnaire et traducteur élémentaire de la LSP. Son caractère d'accessibilité assure son utilisation de la part de tous les citoyens: sourds, malentendants, entendants, aveugles, malvoyants, sourdsaveugles et tous ceux qui présentent un quelconque handicap ou trouble, afin qu'ils puissent avoir accès à la LSP.

Mots-clés : technologie éducative ; langue des signes ; langue des signes panamanéenne ; plates-formes éducatives; outils technologiques; EnSenias. 


\section{Introducción}

En la sociedad del conocimiento, las tecnologías de la información y la comunicación (TIC) son muy conocidas y usadas con frecuencia. Su incursión en el terreno educativo es un hecho, a pesar de que su empleo no esté lo suficientemente deseado y planificado en las programaciones e instituciones educativas, ni tampoco extendido y aprovechado por el conjunto del profesorado y educadores. Esto se produce más por anacronismo de unas por obsolescencia con los tiempos que corren-y reticencia de otros (los “inmigrantes digitales") por falta de formación-, que por falta de interés y competencia de los alumnos (los "nativos digitales naturales").

En la educación, a estas tecnologías se les denomina tecnologías del aprendizaje y la comunicación (TAC), cuando propician el aprendizaje autónomo e individualizado, frente a los tradicionales medios de comunicación, que no permitían la interacción sincrónica (Rodríguez y García, 2012). Y tecnologias online colaborativas (TOC), cuando sirven para el aprendizaje colaborativo, para evitar el aislamiento y fomentar la socialización, enriqueciendo tanto el proceso didáctico como los resultados del mismo (la enseñanza desarrollada y el aprendizaje logrado).

Aunque con cierto desfase en su aplicación en la educación, en la actualidad proliferan programas, aplicaciones, herramientas, servicios disponibles, etc., producto del avance de las TIC y su concreción en TAC y TOC, que están cambiando la forma de vida de personas con discapacidades, al impactar en muchas áreas del conocimiento y al convertirse en poderosos recursos de acción e interacción de dichas personas en la sociedad y con su información (Cabero y Ruiz, 2017). Para quienes padecen sordera, hipoacusia o sordoceguera, "el uso de la tecnología supone una herramienta de gran importancia en cuanto a la eliminación de barreras" (Alaín y Vejarano, 2016, p. 235), de tal suerte que existe una amplia gama de recursos y herramientas que permiten hacer frente a una de sus principales barreras: la comunicación. Dichos recursos incluyen: subtitulación, amplificadores de sonido, editores de imágenes, intérpretes virtuales, programas de reconocimiento de voz que traducen a lengua de signos (LS), que convierten el audio a texto, texto a Ls, etc. Ellos contribuyen a una escuela y una sociedad más inclusivas (Hernández et al., 2019; Rodríguez, 2018).

Cabría concretar un acrónimo a tenor de los anteriores, es decir, como concreción de ellos, que serían las TEC o tecnologías para el empleo de la comunicación, bien porque favorecen, facilitan o mejoran la comunicación en sus distintos lenguajes (oral, escrita, de signos, braille, pictográfica, etc.), bien porque posibilitan, propician o refuerzan su enseñanza y aprendizaje. Costa et al. (2017) se refieren a este tipo de TIC como medio que permite la difusión de diversos signos lingüísticos, lo que coadyuva a la comunicación y la sociabilización. Hernández et al. (2015) añaden que utilizadas "como herramientas para mejorar la comunicación de la población con discapacidad auditiva han permitido el mejoramiento en la calidad de vida de estas personas" (p. 63).

Ahora bien, queda mucho por avanzar con respecto a lo anterior, dado que no todos los contextos disponen de hardware y software apropiados para su LS propia. En otros lugares foráneos, lejanos y cercanos, existen otras plataformas, que se presentan en la Tabla 1, como recursos para la didáctica de la Ls, y que permiten justificar la existencia de una plataforma específica de LSP.

Además, no existe, en Panamá, ninguna herramienta tecnológica dinámica sincrónica con los tiempos actuales, para coadyuvar a la enseñanza-aprendizaje de la LSP y su dinamismo, así como tampoco sobre su uso y perfeccionamiento. Apenas existen conatos de creación de recursos para la Ls propia, como "Mis manos hablan" (Hidalgo, 2011), conjunto de figuras estáticas en formato PowerPoint de LSP, o el obsoleto libro Lengua de señas panameñas (Secretaría Nacional de Discapacidad de Panamá —SENADis-, 2018), en formato pdf (Pimentel et al., 1990). 
Tabla 1 Ejemplos mundiales de aplicaciones didácticas de lenguas de signos

\begin{tabular}{|c|c|c|c|}
\hline Continente & País & Software & Referencia \\
\hline Asia & India & $\begin{array}{l}\text { Conversation of } \\
\text { SL to Speech with } \\
\text { Gestures }\end{array}$ & $\begin{array}{l}\text { Rajaganapathy } \\
\text { ef al. (2015) }\end{array}$ \\
\hline África & Egipto & $\begin{array}{l}\text { An Arabic SL } \\
\text { Recognition } \\
\text { System }\end{array}$ & $\begin{array}{l}\text { Ibrahim et al. } \\
\text { (2017) }\end{array}$ \\
\hline Europa & Varios & Spread Sign & $\begin{array}{l}\text { European sL } \\
\text { Centre (2018) }\end{array}$ \\
\hline Suramérica & Colombia & $\begin{array}{l}\text { Aprendiendo is } \\
\text { colombiana }\end{array}$ & $\begin{array}{l}\text { Hernández et } \\
\text { al. (2016) }\end{array}$ \\
\hline Norteamérica & México & $\begin{array}{l}\text { Aprende señas: lS } \\
\text { mexicana }\end{array}$ & Morales (2018) \\
\hline Centroamérica & Nicaragua & $\begin{array}{l}\text { Aprende y enseña } \\
\text { us nicaragijense }\end{array}$ & Miranda (2018) \\
\hline
\end{tabular}

LS: Lengua de signos; SL: Sign language.

En este punto se inscribe este trabajo, cuyo objetivo tos usuarios potenciales experimentales acerca de la plataforma EnSenias, su pertinencia, accesibilidad y usabilidad, como proceso de validación inicial de la misma.

\section{EnSenias}

La plataforma ${ }^{1}$ fue creada en estrecha colaboración entre el Instituto Panameño de Habilitación Especial, la Universidad Tecnológica de Panamá y las universidades españolas de Granada y Complutense de Madrid.

EnSenias es un recurso tecnológico (caracterizado por ser TEC, TOC, TAC y TIC) cuya finalidad es contribuir a la enseñanza, el aprendizaje, la actualización, el perfeccionamiento, la difusión y el uso de la LSP, en un contexto, Panamá, donde no está generalizado su empleo. La plataforma ha sido ideada y creada como herramienta de aprendizaje y también de enseñanza del vocabulario inicial de lengua de signos panameña (LSP), e igualmente,

1 A la plataforma puede accederse en Ensenias V6 (s. f.).

como diccionario, traductor y preludio del lenguaje escrito. Incluye 1253 signos grabados de 1253 palabras escritas, con sus correspondientes textos escritos y audiodescritos, imágenes representativas y la categoría gramatical de cada palabra.

EnSenias presenta los signos en su generación de movimientos y descripción de los mismos, lo que es posible por su formato de video y audio, y además debidamente actualizados, dado el carácter vivo de la LSP.

La plataforma responde a criterios de:

- Accesibilidad universal: para que resulte accesible a cualquier usuario, con independencia de su (dis)capacidad o dificultad, que desee o precise aprender la LSP, incluso los ciegos y sordociegos, por medio de la audiodescripción y la salida de línea braille. Es decir, no es solo para sordos, sino también para sordociegos, hipoacúsicos, y para otros usuarios oyentes interesados, como familiares, docentes, compañeros, amigos u otros oyentes.

- Empleo con dispositivos diversos: aunque no dispone de versión "App" (por la diversidad de objetos que contiene), está perfectamente adaptada para su uso no solo en computadora, sino también en cualquier dispositivo móvil, y con conexión a internet o sin internet, a través de su descarga en CD o en memoria interna o externa.

- De actualización: al igual que la Ls es una lengua viva, el recurso EnSenias para responder a ella también lo es, permitiendo fácilmente su actualización (incorporación o cambio de palabras en LSP) y, por tanto, su empleo a modo de diccionario de LSP.

- De difusión nacional e internacional: además de crearse como software libre, está desarrollada con una estructura que permite cargar otros videos de otras LS, aprovechando su diseño y, por ende, desarrollo, actividades, imágenes, mensajes escritos, orales y audiodescritos (descripción oral de la información escrita). Pretende 
ser referente para el estímulo e incorporación de otras LS del ámbito americano e iberoamericano.

- Para el desarrollo propio del software se utilizó la metodología de desarrollo de ingeniería de software de Pressman (2002), la cual guía la construcción de la aplicación.

\section{Método}

La investigación sobre la validación de la plataforma EnSenias, de enseñanza y aprendizaje de la LSP, se enmarca como un segundo momento en la metodología de la investigación basada en el diseño (Design-Based Research, IBD), vinculada a la investigación-acción, generada por Lewin (1946) e introducida al terreno de la investigación educativa, entre otros, por metodólogos renombrados, como Anderson y Jones (2000), Carr y Kemmis (1986) y Stenhouse (2007).

La IBD parte del desarrollo multidisciplinar y colaborativo de un recurso que permite dar solución a una problemática identificada, y culmina con su validación en tal escenario, con las percepciones y opiniones expresadas por todos los participantes, en el terreno educativo de manos de usuarios (docentes y discentes) e investigadores. En este sentido, se crea la plataforma EnSenias para dar solución a la problemática de la falta de una plataforma propia para la LSP, y se hace la validación que aquí se presenta.

El diseño de la IBD proviene de la ingeniería y la programación, así como de otras ciencias aplicadas (de Benito y Salinas, 2016). Se enmarca dentro de los diseños no experimentales, exploratorios, transversales, de tipo aplicados y de enfoque mixto, de base eminentemente cualitativa, pero con tratamiento estadístico de datos, una vez codificadas las respuestas ofrecidas por los participantes en la investigación. De esta manera, esta investigación cumple con tales características, y el tratamiento estadístico se hizo una vez que fueron codificadas las respuestas ofrecidas por los participantes en la investigación y transformadas en frecuencias positivas o negativas de validación de EnSenias.

\section{Técnica y procedimiento de recogida de datos}

Se trabajó mediante la técnica de campo de recogida de datos de entrevista grupal semiestructurada, en concreto, los grupos focales (focus groups), al ser la más específica de entre todas las modalidades de entrevistas para focalizar una cuestión determinada (Ramírez, 2015), con la ayuda de un guion de preguntas como instrumento.

Tras su conformación, los grupos fueron reunidos en una sesión plenaria, donde se les presentó la aplicación EnSenias en su versión inicial y se trabajó con ella para su mejora. En una reunión posterior global, que incluyó el reconocimiento a los participantes y la relevancia de sus aportaciones, durante una hora se les ilustró sobre la versión definitiva y su uso, y se respondieron las dudas planteadas. Seguidamente, se indicó una serie de ejercicios prácticos que cada participante debía realizar por su cuenta durante un tiempo máximo de una hora, propia para garantizar la experimentación de la aplicación. Al final, fueron invitados a otras sesiones, esta vez por colectivos específicos, a efectuar en la semana siguiente.

En estas últimas reuniones se procedió a la realización de las entrevistas grupales propiamente dichas o grupos focales. Allí se formuló una serie de preguntas sobre la aplicación, con el objeto de suscitar el debate de los miembros de cada colectivo en sesiones separadas, enriquecido por el propio grupo, de tal suerte que el total (grupo) resultara más que la suma de las partes (individualidades). Las sesiones fueron grabadas en audio, con la debida autorización escrita de los entrevistados, para su ulterior trascripción textual.

\section{Participantes del estudio de validación de la plataforma}

Se constituyó una docena de grupos de naturaleza muy diferente, en total 57 participantes, vinculados con el aprendizaje, la enseñanza y el empleo de la LSP (véase Tabla 2). 
Tabla 2 Descripción e identificación de los grupos focales del estudio

\begin{tabular}{|c|c|c|c|c|}
\hline & & Grupos focales: naturaleza de los colectivos & $\mathbf{N}$ & Código \\
\hline 1 & Alumnos & Sordos que aprenden y usan la LSP (AS) & 6 & $\mathrm{ASI} / \ldots / \mathrm{AS} 6$ \\
\hline 2 & & Compañeros de alumnos que usan la LSP (AC) & 3 & $\mathrm{ACl} / \mathrm{AC2} / \mathrm{AC} 3$ \\
\hline 3 & Progenitores (pad & dres y madres de niños con sordera) (PS) & 5 & PS1 $/ \ldots /$ PS5 \\
\hline 4 & Intérpretes que $\dagger$ & trabajan con alumnos que usan la LSP (IL) & 4 & $\mathrm{ILI} / \ldots / \mathrm{AL} 4$ \\
\hline 5 & Docentes & Especialistas de alumnos con sordera (DS) & 7 & DS1/.../DS7 \\
\hline 6 & & Generalistas, no especialistas de aula, con alumnos con sordera (DG) & 6 & $\mathrm{DGl} / \ldots / \mathrm{DG} 6$ \\
\hline 7 & & Especialistas en sordociegos que precisan audiodescripción (DC) & 2 & $D C 1 / D C 2 / D C 3$ \\
\hline 8 & & Universitarios expertos en atención a la discapacidad (DU) & 2 & DU1/DU2 \\
\hline 9 & & Universitarios expertos en discapacidad auditiva (DE) & 4 & $\mathrm{DEl} / \ldots / \mathrm{DE} 4$ \\
\hline 10 & Representantes & De la Asociación de Sordos de Panamá (RS) & 6 & $\mathrm{RS} 1 / \ldots / \mathrm{RS} 2$ \\
\hline 11 & & De la Asociación de Sordos Cubana (RC) & 4 & $\mathrm{RCl} / \ldots / \mathrm{RC} 4$ \\
\hline 12 & & Del Instituto Nacional de Sordos de Colombia (RI) & 8 & $\mathrm{RI} / \ldots / \cdot \mathrm{RI}$ \\
\hline Total & & & \multicolumn{2}{|c|}{57 participantes } \\
\hline
\end{tabular}

Siguiendo las indicaciones de Sampieri et al. (2010), el tamaño de los grupos debe oscilar entre tres y diez personas, y así se hizo (salvo en dos colectivos, por imponderables de última hora de las agendas de los participantes), para garantizar un debate suficientemente enriquecido y que a la vez resultara operativo para la participación de todos, más un moderador (e incluso dos, en ocasiones), que realizó las preguntas y orientó la discusión, tras crear el ambiente adecuado, es decir, relajado e informal, que invitara a la participación libre y cómoda de cada miembro. Cuando fue necesario, se incorporó un intérprete de LSP, para posibilitar una comunicación fluida.

Al tratarse de una investigación con personas, algunos alumnos menores de edad, se hizo la solicitud institucional (Instituto Panameño de Habilitación Especial) e individual de directrices éticas de información, voluntariedad, tratamiento y anonimato de los participantes, aprobadas por la Universidad de Granada (Universidad de Granada. Vicerrectorado de Investigación y Transferencia, s. f.).

\section{Análisis de datos}

Una vez efectuada la transcripción completa de las declaraciones de los participantes sobre las cuestiones ya enunciadas, se procedió, en un primer momento, a una revisión inicial probatoria, por el grupo de codificadores, de las declaraciones de los grupos focales. En un segundo momento, se efectuó el ensayo con las categorías preestablecidas, a partir de algunas de las declaraciones, por el mismo grupo de expertos, de tal suerte que de su acuerdo y consenso (en torno al $95 \%$ final) surgieron las categorías definitivas de agrupamiento de declaraciones, con sus códigos correspondientes, que se muestran en la Tabla 3. Con ello se culminó el proceso previo del análisis de contenido, mediante el establecimiento de categorías apriorístico.

En tal experimentación, teniendo en cuenta que se trata de reducir la información a categorías y posibles metacategorías, y de acuerdo con los postulados de investigación sobre el análisis de contenido, se estimó que las categorías no superaran la docena, sin perjuicio de que posteriormente, en el análisis global, pudieran inevitable añadirse algunas más.

En suma, se determinaron cuatro metacategorías: 1) valoración general de la plataforma, 2) usabilidad [general] de la plataforma, 3) mejoras [de la plataforma], y 4) limitaciones insalvables de la plataforma. Dentro de ellas, tuvieron cabida las categorías concretas, con sus códigos correspondientes, contempladas en la Tabla 3. 
Tabla 3 Metacategorías, categorías y códigos utilizados para registro de los grupos focales

\begin{tabular}{|c|c|c|c|c|}
\hline & \multirow{2}{*}{\multicolumn{2}{|c|}{$\begin{array}{l}\text { (Meta)categorías. } \\
\text { Enunciado básico y su código neutro }\end{array}$}} & \multicolumn{2}{|c|}{ Código } \\
\hline & & & \multirow{2}{*}{$\begin{array}{l}+ \\
\mathrm{VGP}+\end{array}$} & \multirow{2}{*}{$\frac{-}{-}$} \\
\hline 1 & \multicolumn{2}{|c|}{ Valoración general de la plataforma (VGP) } & & \\
\hline 2 & \multirow{5}{*}{$\begin{array}{l}\text { Usabilidad [general] de la } \\
\text { plataforma (UGP) }\end{array}$} & para aprender la LSP (UPA) & UPA + & UPA- \\
\hline 3 & & para enseñar la LSP (UPE) & UPE+ + & UPE- \\
\hline 4 & & como diccionario de palabras (UPD) & UPD + & UPD- \\
\hline 5 & & como traductor de palabras (UPT) & UPT+ & UPI- \\
\hline 6 & & para aprender la lectoescritura (UPL) & UPL+ & UPL- \\
\hline 7 & \multirow[t]{3}{*}{ Mejoras [de la plataforma] } & de palabras contenidas en la plataforma (MPP) & MPP + & MPP- \\
\hline 8 & & de la estética de la plataforma (MEP) & MEP + & MEP- \\
\hline 9 & & de la funcionalidad de la plataforma (MғF) & MFP + & MFP- \\
\hline 10 & \multicolumn{2}{|c|}{ Limitaciones insalvables de la plataforma (LIP) } & LIP+ & LIP- \\
\hline
\end{tabular}

LSP: Lengua de signos panameña.

El proceso de análisis de contenido consistió en una categorización a posteriori, al no disponer de un modelo teórico que orientara el proceso de categorización apriorística (Bardin, 1996). Esto dejaba abierta la puerta a la incorporación posterior de nuevas categorías en el análisis de contenido profundo y exhaustivo de las declaraciones, una vez que se procediera a su categorización, lo cual no ocurrió.

Fueron detalladas, por consenso del grupo de profesionales codificadores de la investigación, el sentido de cada categoría y su signo, tomando en consideración para ello que respecto de la valoración y el uso de la plataforma, el signo positivo indica que son óptimos, y el negativo, pésimos, mientras que acerca de la mejora, el signo positivo expresa que las mejoras son susceptibles de acometerse (críticas constructivas) y el signo negativo indica que no es posible acometerse las mejoras señaladas como necesarias o aconsejables (críticas insalvables).

La categorización definitiva, de tipo inductiva, derivada del proceso codificador antes descrito: previo, inicial o en primer plano global - descrito por Coleman y Unrau (2005) — , y continuado mediante una codificación posterior central o en segundo plano — siguiendo a Grinnell (1997) —, se realizó en un tercer momento del estudio, una vez que se contaba con la transcripción de las declaraciones.
Tres investigadores realizaron por separado la categorización, mediante una sencilla tabla de Excel ${ }^{\circ}$, analizada con el programa estadístico o complemento EZanalyze ${ }^{\circ}$, integrado en el programa Excel $^{\triangleright}$, con lo que se obtuvo el grado de acuerdo entre expertos. El valor de acuerdo entre las categorías indicadas, calculado por categorías, osciló entre el 86 y el $98 \%$, lo que computó un acuerdo global interinvestigadores en torno al $93 \%$, que se estima alto. No se añadieron nuevas categorías.

Posteriormente, se procedió al análisis de contenido propiamente cualitativo, según las exigencias propuestas por Bardin (1996) y aceptadas por la comunidad de investigadores (Hernández et al., 2010), en el sentido de la interpretación de las declaraciones aportadas por los intervinientes en los diferentes grupos focales, ahora consideradas "datos de la investigación", agrupados y reducidos en metacategorías.

La agrupación de las declaraciones por categorías y estas, a su vez, por metacategorías propició, además del análisis correspondiente de tipo ideográfico, el recuento frecuencial y porcentual de las declaraciones por participantes, colectivos y totales. Tras este básico análisis de tipo cuantitativo, se procedió a la esencia de la investigación, es decir, al análisis cualitativo de contenido, de mayor complejidad. 
La inclusión de análisis cuantitativos dentro del propósito esencial de esta investigación, correspondiente conun análisis de tipo cualitativo, responde a las tendencias actuales de análisis mixtos o Mixed Method Research (Rodríguez y Caurcel, 2019).

Para el desarrollo de los análisis se emplearon programas informáticos. Preliminarmente, se empleó el programa Transcriber ${ }^{\oplus}$, para transcribir las informaciones de las entrevistas al formato digital. Respecto del análisis cualitativo de contenido, para la reducción de información y su categorización y metacategorización se empleó el programa NVivo ${ }^{\circ}$ versión 11, de QSR International, lo que permitió la reducción y la agrupación de declaraciones por colectivos y categorías. Posibilitó, a su vez, un recuento de categorías positivas y negativas, relativas y acumuladas, lo que se aprovechó para recontar y agrupar estos datos numéricos.

Estos datos, incorporados en tablas correspondientes del programa Excel $^{\circ}$, fueron trabajados mediante el paquete estadístico SPSS $^{\oplus}$ versión 24.0, de IBM ${ }^{\oplus}$, para sus análisis descriptivos y de dispersión básicos, y en su caso, profundizar en la estadística inferencial más compleja. Es decir, en primer lugar, se hicieron sencillos cálculos estadísticos de frecuencias relativas, acumuladas y totales, de tipo porcentual, de tendencia central $($ medias $=\overline{\mathrm{x}}) \mathrm{y}$ de dispersión $($ desviación típica $=s)$. En segundo lugar, dada la naturaleza de los grupos y su tamaño, no cabía realizar otros tipos de análisis paramétricos complejos de tipo inferencial, pero sí no paramétricos. En tal sentido, se contrastaron las diferencias entre grupos focales y sus medias y varianzas, mediante la prueba $U$ de Mann Whitney (U), junto a la prueba post-HSD de Tukey (HSD) y de Bonferroni (B), para identificar, en su caso, entre cuáles de los grupos se presentaban las diferencias. Por último, para las relaciones entre respuestas y categorías, se recurrió al coeficiente de correlación rho de Spearman $(r)$. Todo ello a un nivel de significación $(p)$ menor que el 0,05, es decir, asumiendo un porcentaje de error en la generalización del $5 \%$.

\section{Resultados}

Siguiendo la metodología, tras la creación de EnSenias, se realizó la validación de la misma, a través de la percepción y la opinión de los usuarios sobre la plataforma, cuyos resultados, de acuerdo con las categorías advertidas resultantes de las declaraciones y los debates de los entrevistados, así como por sus frecuencias de aparición y los análisis estadísticos permitidos, se presentan a continuación, según las metacategorías determinadas.

\section{Valoración de EnSenias para los diversos colectivos y usos}

Para comenzar con el diálogo grupal de modo general, se comenzó preguntando por sus pareceres acerca de la aplicación EnSenias, tras su empleo. De sus respuestas, surgió la categoría "Valoración general de la plataforma” (VGP), que obtuvo una valoración positiva por todos los grupos y participantes, con mayor o menor énfasis, junto a una nula valoración negativa (véase Figura 1).

Algunos se expresan con gran euforia e incluso reconocimiento, como los alumnos que signan la palabra "gracias" al principio y al final de la entrevista, intercalada por la indicación de "sois unos ángeles" de uno de ellos (AS1), al igual que los padres que se lamentan por no "haber tenido este programa antes" (PS3) y que manifiestan que se tiene que "usar desde ya con el apoyo institucional" (RS2). Otros con recelo de que, aun resultando "pertinente" (IL2) y "muy necesaria" (IL1), no "puede sustituir al comunicador [adulto que enseña y comunica mediante LS]" (IL4).

En suma, las valoraciones positivas (VGP+) resultaron muy frecuentes y las negativas testimoniales, de tal suerte que la media total de aparición por individuo es 2,46 veces en positivo (un total de 140 respuestas), frente a 0,04 en negativo (apenas dos respuestas), como se aprecia en la Figura 1.

Además, las diferencias entre las valoraciones por los distintos grupos no alcanzaron la significación 


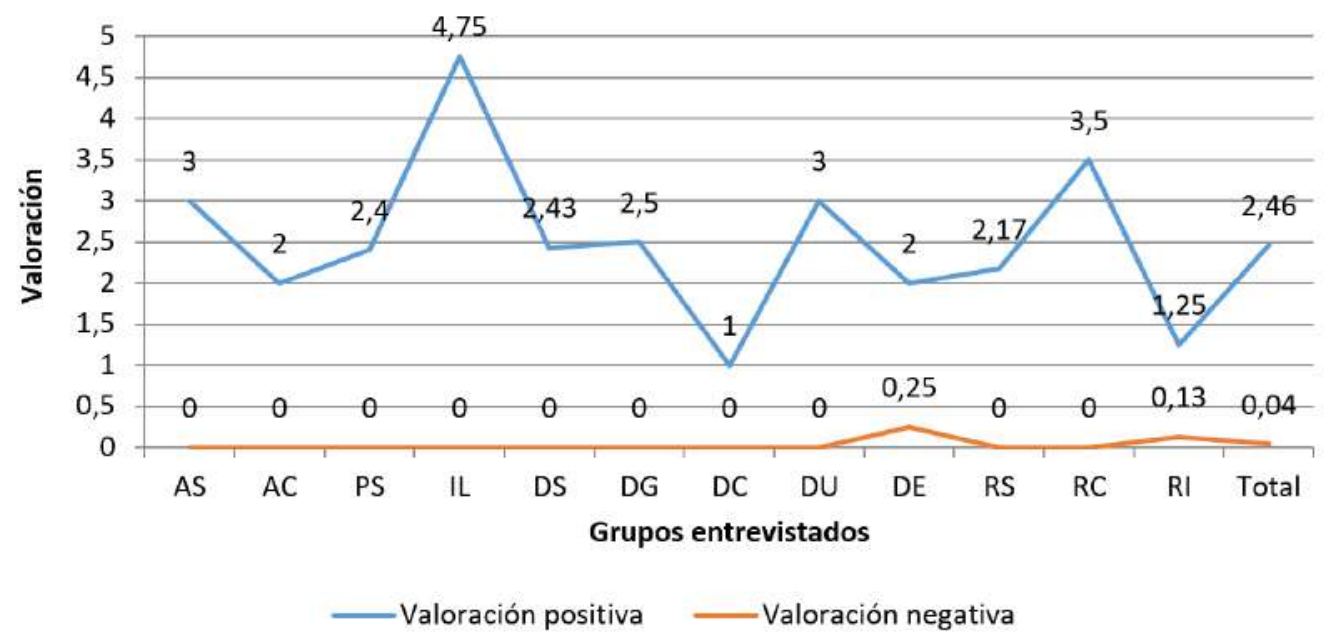

Figura 1 Medias de la categoría "Valoración general de plataforma". Grupos: AS: Alumnos sordos; AC: Alumnos compañeros de alumnos que pudieran usan la lengua de signos panameña (LSP); PS: Progenitores (padres y madres de niños con sordera); IL: Intérpretes que trabajan con alumnos que usan la LSP; DS: Docentes especialistas de alumnos con sordera; DG: Docentes generalistas, no especialistas de aula, con alumnos con sordera en sus aulas inclusivas; DC: Docentes especialistas en sordociegos que precisan audiodescripción; DU: Docentes universitarios expertos en atención a la discapacidad; DE: Docentes universitarios expertos en discapacidad auditiva; RS: Representantes de la Asociación de Sordos de Panamá; RC: Representantes de la Asociación de Sordos Cubana; RI: Representantes del Instituto Nacional de Sordos de Colombia.

estadística $(p=0,09)$ al aplicar la prueba $U$ de Mann Whitney $(\mathrm{U}=1,87)$ sobre las frecuencias absolutas (restando las respuestas positivas y negativas de la categoría), dado que todos los emparejamientos posibles intergrupos contemplaban datos aproximados positivos de valoración y prácticamente carecían de negativos. Luego solo se percibe un grupo homogéneo que reconoce y valora la plataforma EnSenias; por tanto, la categoría queda validada cualitativa y cuantitativamente, como se observa en la Figura 1.

\section{Usabilidad [general] de la plataforma}

De las declaraciones sucesivas a la preliminar VGP se desprende que la óptima valoración general se debe a la percepción sobre la metacategoría "Usabilidad [general] de la plataforma" (UGP) y sus cinco categorías constituyentes (véase Figura 2).

La valoración ha sido notablemente positiva; de hecho, apenas han aparecido 4 respuestas negativas: "se requiere empoderar la cultura sorda, no solo la Ls" (DE2) y la "morfosintaxis" (IL4), lo cual "requiere del instructor de LS"(IL1), frente a las 255 positivas: "recurso adecuado al modo de ser de los jóvenes sordos" (IL1) y "ciegos y sordociegos" (DC1), "muy necesario [EnSenias] para padres" (PS2), que requiere inmediatamente del "concurso de instituciones para empezar a usarla cuanto antes" (DS3), "puesto que es fabulosa para aprender, enseñar y dominar la LSP por encima del actual manual de LSP" (RS4).

Profundizando en las más frecuentes, es decir, en las positivas, no han sido distribuidas de manera similar entre las distintas categorías, como se observa en la Figura 2. En efecto, algunas categorías sobre "usabilidad" aparecen con bastante intensidad. La que más, la "Usabilidad de la plataforma para aprender" (UPA), ha sido la mejor valorada, mostrando alto grado de acuerdo de su potencial "para aprender todas las palabras de la LSP" (ES1), "para sordos y oyentes que la precisen” (IL3) y "de manera oral también para sordociegos con restos 


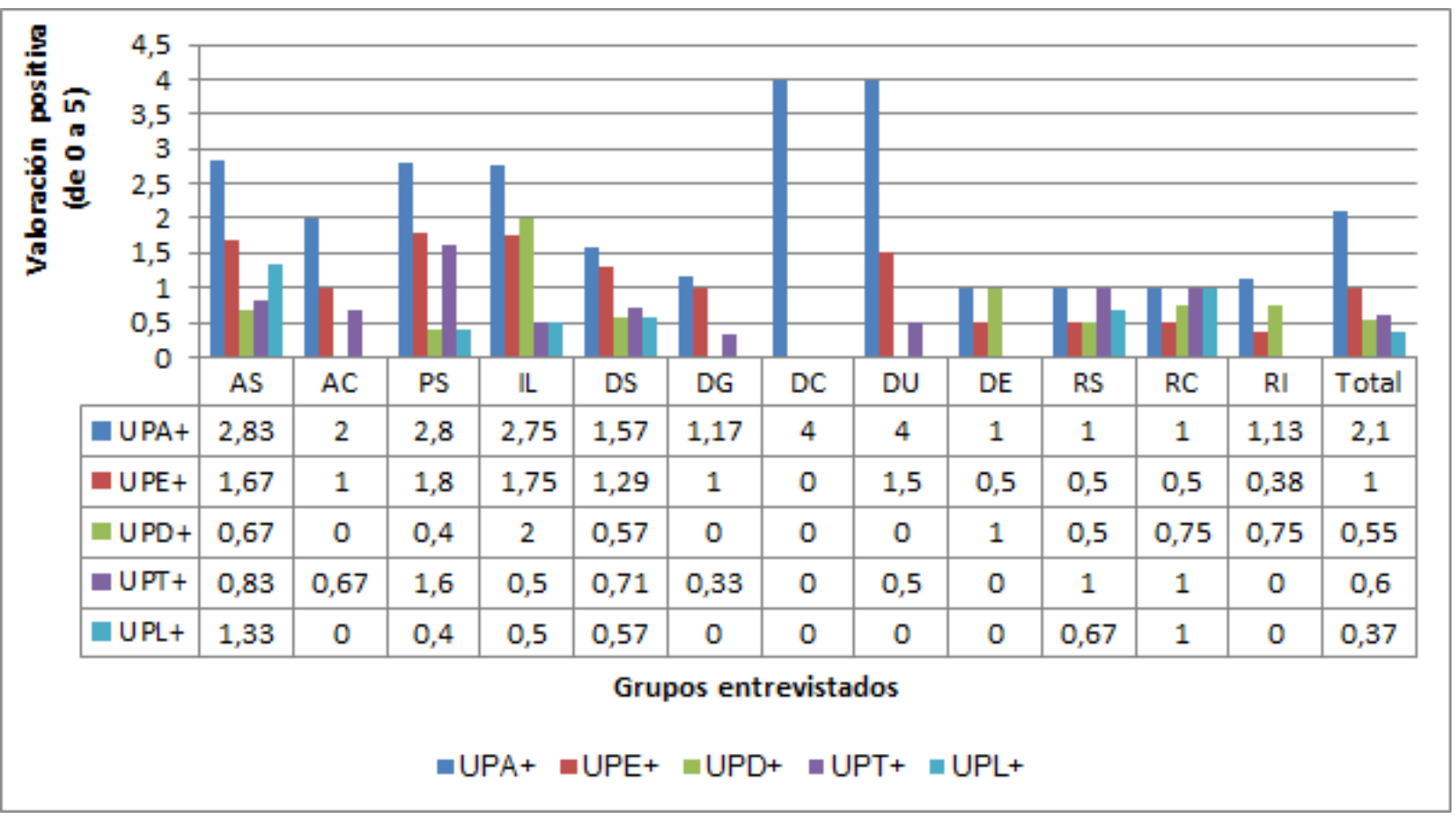

Figura 2 Medias de la metacategoría "Usabilidad [general] de la plataforma” y sus categorías constituyentes. Grupos: UPA: Usabilidad para aprender la lengua de signos panameña (LSP); UPE: Usabilidad para enseñar la LSP; UPD: Usabilidad de la plataforma como diccionario de palabras; UPT: Usabilidad como traductor de palabras; UPL: Usabilidad para aprender la lectoescritura. Colectivos: AS: Alumnos sordos; AC: Compañeros de alumnos que usan la LSP; AS: Alumnos sordos que aprenden y usan la LSP; DC: Docentes especialistas en sordociegos que precisan audiodescripción; DE: Docentes universitarios expertos en discapacidad auditiva; DG: Docentes generalistas, no especialistas de aula, con alumnos con sordera en sus aulas inclusivas; DS: Docentes especialistas de alumnos con sordera; DU: Docentes universitarios expertos en atención a la discapacidad; IL: Intérpretes que trabajan con alumnos que usan la LSP; PS: Progenitores (padres y madres de niños con sordera); RC: Representantes de la Asociación de Sordos Cubana; RI: Representantes del Instituto Nacional de Sordos de Colombia; RS: Representantes de la Asociación de Sordos de Panamá.

auditivos" (DC1). La media de aparición de esta respuesta por individuo ha sido de $\bar{x}=2,10$ veces, con una dispersión relativa de $s=0,51$.

También, dentro de esta alta prevalencia, se sitúa la "Usabilidad de la plataforma para enseñar" (UPE), con todas las apreciaciones positivas, aunque menos fuerte que la anterior $(\bar{X}=1)$, y con dispersión similar $(s=0,63)$, salvo una: "no basta con un recurso, por bueno que sea para enseñar; se requiere[n] habilidades docentes y trato con el sordo" (IL4).

Ni en una ni en otra categoría se constatan diferencias entre los colectivos, según la $U$ de Mann Whitney $\left(\mathrm{U}_{\mathrm{UPA}}=4,84 \mathrm{y} \mathrm{U}_{\mathrm{UPE}}=0,96\right)$, que alcancen significación estadística preestablecida $(p=0,6 \mathrm{y}$ $p=0,49$, respectivamente); luego todos los grupos muestran su apuesta por ambas usabilidades. Además, entre ellas obtienen un alto emparejamiento, tras aplicar a sus frecuencias de aparición el coeficiente de correlación de Spearman, con un valor positivo y elevado de relación $\left(r_{\text {UPA-UPE }}=0,82\right.$; $p=0,00)$. Aún más, son las responsables de la buena VGP, categoría anterior, dado que también con ella resultan intensamente relacionadas las respuestas, según los valores de $r$ ho de Spearman $\left(r_{\mathrm{VGP}-\mathrm{UPA}}=0,79, p=0,04, \mathrm{y} r_{\mathrm{VGP}-\mathrm{UPE}}=0,75, p=0,05\right)$. Puede afirmarse, por tanto, la usabilidad de la plataforma, tanto para aprender (UPA) como para enseñar (UPE). De ahí su valoración general de la plataforma (VGP). 
En otro bloque se sitúan las categorías "Usabilidad de la plataforma como diccionario de palabras" (UPD) y "Usabilidad como traductor" (UPT), con unas frecuencias medias de aparición de $\bar{x}=0,55$ y 0,60 , respectivamente, esto es, cerca de una aparición por cada dos participantes, si bien con dispersiones relativamente altas $(s=0,58$ y 0,64 , respectivamente). Ello se debe a la existencia de diferencias entre individuos e, incluso, entre colectivos, como ha puesto de manifiesto el cálculo de la $U$ de Mann Whitney $\left(\mathrm{U}_{\mathrm{UPD}}=2,99, p=0,009 \mathrm{y}\right.$ $\left.\mathrm{U}_{\mathrm{UPT}}=1,06, p=0,041\right)$.

Por una parte, respecto de su UPD, el consecuente cálculo post hoc aclara las diferencias entre ellos y dibuja dos subgrupos heterogéneos: uno para $8 \mathrm{de}$ los grupos focales $(66,67 \%)$ : de alumnos sordos (AS), sus padres (PS), los intérpretes (IL), los docentes de sordos y expertos en discapacidad auditiva (DS y DE) y los representantes de diferentes contextos (RS, RC y RI) que sí contemplan este uso de la plataforma "como diccionario para perfeccionar signos” (ES3), “buscar palabras nuevas” (IL1) e "introducir nuevos signos" (RS4); y otro para los demás (33,33\%): alumnos oyentes (AC), docentes generalistas (DG), docentes ciegos (DC), docentes universitarios (DU) que no resaltaron tal uso. Es decir, cabría concluir que los colectivos directamente vinculados con la LS (los 8 primeros) sí perciben el valor de la plataforma "como diccionario", a diferencia de los menos vinculados (los 4 segundos). Además, se observa cierto vínculo (relación media, significativa y directa) con respecto a las anteriores categorías (VGP, UPA y UPE) con la UPD, según el coeficiente de correlación de Spearman $\left(r_{\text {UPD-VGP }}=0,46, p=0,040 ; r_{\text {UPD-UPA }}=0,49\right.$, $\left.p=0,050, \mathrm{y} r_{\text {UPD-UPE }}=0,54, p=0,000\right)$.

Por otra parte, respecto de la "Usabilidad de la plataforma como traductor" (UPT), igualmente se manifestaron diferencias significativas después del cálculo estadístico entre unos grupos y otros, lo cual llevó a establecer también dos grupos (tras el cálculo de HSD y B): el de colectivos que sí contemplaron este uso (10 del total: 83,33\%) de EnSenias para "saber qué quieren decir" (PS5) los usuarios de LSP y “mostrárselo a los demás” (AC), entre los que están: el de alumnos sordos y sus compañeros (AS y AC), sus padres (PS), los intérpretes (IL), los docentes de sordos, generalistas y universitarios (DS, DG y DU) y los representantes (RS, RC y RI); a diferencia de los demás (2 colectivos: $16,67 \%)$, que le resta fuerza a este uso: docentes ciegos y expertos en sordera (DC y DE). Igual que la anterior, aparece relacionada con otras categorías $\left(r_{\text {UPT-VGP }}=0,84, p=0,004 ; r_{\text {UPT-UPA }}=0,64\right.$, $\left.p=0,044, \mathrm{y} r_{\text {UPT-UPE }}=0,71, p=0,032\right)$.

Puede concluirse, aun sin la contundencia de las anteriores, con una validación tímida de estas categorías de "Usabilidad [general] de la plataforma" como "diccionario" (UPD) y como "traductor" (UPT). Al contrario acontece con la categoría "Usabilidad de la plataforma para aprender la lectoescritura" (UPL), que no alcanza frecuencias de aparición positiva $(f=24)$ para su validación (aunque se reconozca, en algunos casos, que "al aparecer la palabra y su signo se aprenden ambos” - ES1-), con una media de aparición por individuo de $\bar{x}=0,37$, es decir, resaltada por 1 de cada 3 participantes, $y$ una dispersión de $s=0,66$.

Existen, también, diferencias entre grupos $\left(\mathrm{U}_{\mathrm{UPL}}=5,09, p=0,05\right)$, pero no se dibujan claramente subconjuntos diferentes, dadas las bajas frecuencias advertidas, y solo la mitad de los grupos resaltan esta usabilidad, lo cual impide que se pueda considerar esta categoría como validada. Tampoco resulta relacionada con las anteriores, dado el bajo valor relacional $(r<0,04)$ o su grado de significación $(p>0,05)$.

\section{Mejoras salvables y limitaciones insalvables de la plataforma EnSenias}

No menos importantes en el diseño de investigación seguido y para los objetivos de este trabajo son las críticas constructivas vertidas a la plataforma, que pueden visualizarse globalmente en la Figura 3.

Por una parte, las "Mejoras [de la plataforma]" han sido incorporadas en sucesivas versiones de 


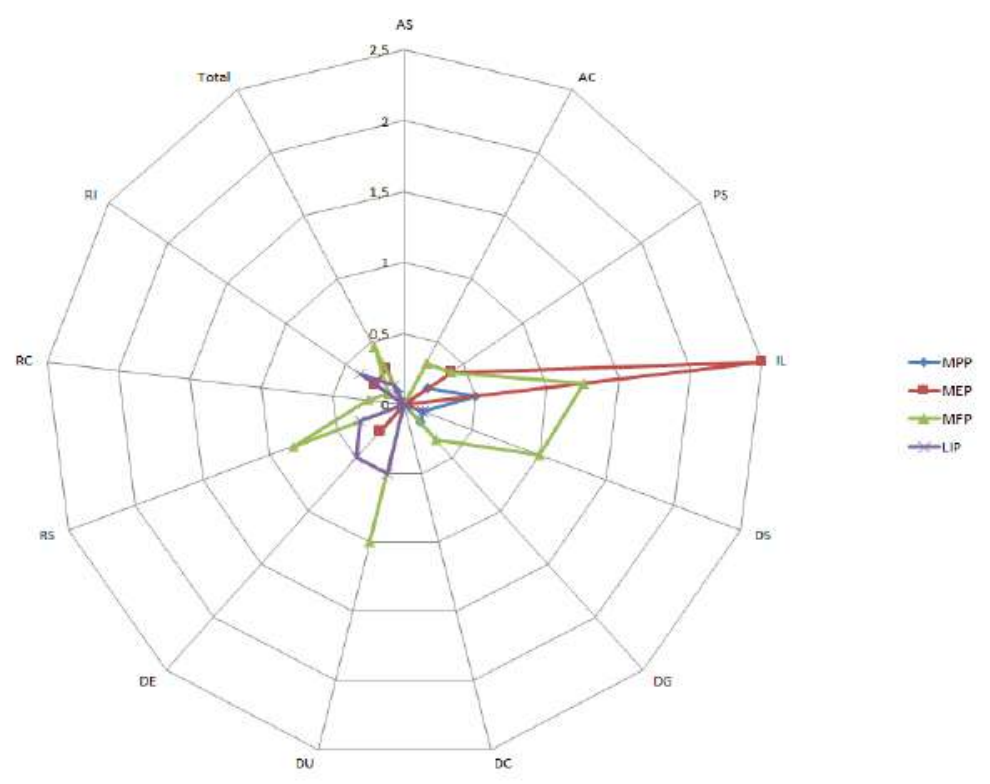

Figura 3 Medias de las metacategorías "Mejoras [de la plataforma]". Categorías: MPP: Mejoras de palabras contenidas en la plataforma; MEP: Mejoras de la estética de la plataforma; MFP: Mejoras de la funcionalidad de la plataforma; LIP: Limitaciones insalvables de la plataforma. Grupos: AS: Alumnos sordos; AC: Compañeros de alumnos que usan la LSP; AS: Alumnos sordos que aprenden y usan la LSP; DC: Docentes especialistas en sordociegos que precisan audiodescripción; DE: Docentes universitarios expertos en discapacidad auditiva; DG: Docentes generalistas, no especialistas de aula, con alumnos con sordera en sus aulas inclusivas; Ds: Docentes especialistas de alumnos con sordera; DU: Docentes universitarios expertos en atención a la discapacidad; IL: Intérpretes que trabajan con alumnos que usan la LSP; PS: Progenitores (padres y madres de niños con sordera); RC: Representantes de la Asociación de Sordos Cubana; RI: Representantes del Instituto Nacional de Sordos de Colombia; RS: Representantes de la Asociación de Sordos de Panamá.

EnSenias, hasta la versión 6 definitiva, y se refieren a las categorías: "Mejoras de palabras contenidas en la plataforma” (MPP), "Mejora de la estética de la plataforma” (MEP) y "Mejoras de la funcionalidad de la plataforma” (MFP)”. Resalta la relativa aparición de la $\operatorname{MFP}(\overline{\mathrm{X}}=0,46 ; s=0,44)$, en torno principalmente a la incorporación de "la morfosintaxis de la LSP" (RI2), ya que mediante quizá la introducción de las oraciones "puede dar más juego" (IL2), así como de "actividades de trabajo" (DS3) de autoevaluación y la "utilización de lectura mecanizada más familiar para ciegos” (DC1); y todo ello "no solo a través de internet", sino también en otros formatos (RS3). Al contrario, escasean las declaraciones del resto de categorías, denotando una prevalencia baja:

- $\bar{x}=0,10$ para la MPP, por la existencia de "sinónimos para algunas palabras" (IL5) e “introducción de la palabra 'internet'” (DE2), así como un debate surgido en torno al "cambio de la categoría 'vestido' por la de 'habilidades de vida diaria'" (DE3), cambio que no le pareció "oportuno" a otro participante (D1).

- $\overline{\mathrm{x}}=0,28$ para la MEP, sobre la variación de figuras representativas: "dibujos" (PS4), así como el aumento del "tamaño completo de pantalla" (IL4) para la reproducción de los signos y del "contraste de figura y fondo" (IL1).

Dada la expresión de estas ideas por solo algunos participantes, la dispersión es alta en las tres categorías anteriores $(s=0,95,1,21$ y 1,14 , respectivamente). Pero las variaciones grupales son significativas en el caso de la MFP $\left(\mathrm{U}_{\mathrm{MFP}}=3,103\right.$, $p=0,008)$, que distingue dos subgrupos: uno mayoritario ( 9 de los 12: $75 \%$ ), que advierte mejoras 
funcionales para la plataforma: alumnos compañeros (AC), padres de sordos (PS), intérpretes (IL); docentes de sordos, generalistas y universitarios (DS, DG y DU), y los representantes de sordos (RS, RC y RI); y otro grupo minoritario (3 grupos: $25 \%$ ), que no expresa mejoras: alumnos sordos (AS), docentes ciegos (DC) y docentes expertos (DE).

Para la MPP no existen diferencias entre grupos $\left(\mathrm{U}_{\mathrm{MPP}}=1,063, p=0,413\right)$, dadas las bajas frecuencias de aparición. Y para la MEP, solo el grupo IL obtiene diferencias $\left(\mathrm{U}_{\mathrm{MFP}}=3,103, p=0,008\right)$ en el cálculo estadístico HSD $(p<0,05)$ respecto del resto.

Por último, no existe correlación entre estas categorías entre sí ni con respecto a las anteriores, según los coeficientes de Spearman calculados. Todo lo anterior induce a pensar que las "Mejoras propuestas" no restan ni un ápice a la "Valoración general de la plataforma" ni a la "Usabilidad [general] de la plataforma", metacategorías anteriores, ni son muchos los que plantean "Mejoras [de la plataforma]", categoría actual, sino más bien puntuales, como se observa en la Figura 3.

Por otra parte, las críticas no resueltas en versiones futuras, denominadas "Limitaciones insalvables de la plataforma (LIP)", alcanzan una media insignificante de $\bar{x}=0,15$ (véase Figura 3), con una dispersión elevada $(s=1,39)$, y aluden principalmente a: 1) "la necesidad de incorporar lectura y escritura” (DE3), lo cual requiere otro trabajo complementario, pero diferente; 2) la configuración de la plataforma "a través de una App" (RS3), inviable por la cantidad de objetos - 1253 videos, audios, textos, imágenes, etc.- (aunque sí permite su visualización a través de tecnología móvil), y 3) la consideración de "los rasgos distintivos de los signos panameños según zonas” (DE4), que se antojan casi individuales.

Solo los grupos de docentes universitarios y expertos (DU y DE) y algunas asociaciones de representantes (RS y RI), es decir, 4 grupos $(33,33 \%)$, han manifestado estas limitaciones de manera testimonial, aunque ello los diferencia significativamente $\left(\mathrm{U}_{\mathrm{IIP}}=0,379 ; p=0,024\right)$ del resto de colectivos, los 8 restantes $(66,67 \%)$, que no las han contemplado. No existe relación con el resto de categorías $(r<0,4$ o $p>0,05)$.

\section{Discusión y conclusiones}

No hay plataformas ni herramientas informáticas en el contexto de Panamá para el que EnSenias ha sido creada; apenas existen algunos conatos de presentación estática de signos (Alaín y Vejarano, 2016), aunque sí las hay en otros contextos (Cfr. Alaín, 2019); por lo tanto, cobra sentido el esfuerzo realizado. No obstante, algunas herramientas similares en otros contextos comenzaron a desarrollarse a finales del siglo pasado y especialmente a principios del presente. Algunas de ellas se limitan a la tarea de enseñanza y aprendizaje del alfabeto en LS, como el "Pequeabecedario" de la LS española (Cfr. González y Sola, 2006), o la de Chacón et al. (2014), con la Ls ecuatoriana.

Otras contienen la reproducción signada en LS española de palabras, como "Intelex" o “ $\mathrm{A}$ signar!” (Cfr. González y Sola, 2006), que, como en el caso presentado, además se orientan a la enseñanzaaprendizaje. La primera, además, se acompaña de la descripción oral de posiciones y movimientos. $\mathrm{Al}$ ser tradicionales, aparecieron en formato $\mathrm{CD}$ Rom y con ayudas en formato físico.

En los últimos tiempos han emergido variadas herramientas tecnológicas más interactivas y modernas para propiciar el aprendizaje de la Ls y para coadyuvar su enseñanza, y no necesariamente en formato físico, sino online, lo que ha extendido su visualización y sus posibilidades de empleo. Entre las más actuales y cercanas a la que se presenta están las de Garza y Monsivais (2015) y Miranda (2018) para México; Hernández et al. (2016) para la Ls colombiana; Miranda (2018), para la Ls nicaragüense, y la de la Universidad Nacional de Costa Rica (2018), para su LS nacional. Una revisión de otras anteriores y de otros contextos puede hallarse en Hernández et al. (2015) y Alaín (2019).

Lo cierto es que la mayoría de ellas provienen del ámbito de la ingeniería de la programación, sin

\section{5}


vinculación ni colaboración desde su creación con los campos educativo y comunicativo, y aún menos con los usuarios de la plataforma. En esta ocasión, se ha trabajado en equipo multidisciplinar, tanto con programadores como con expertos en pedagogía y comunicación e investigación educativa, y además con demandantes, usuarios e instructores de la LS. Ello ha propiciado que, por el propio diseño de IBD, se hayan culminado hasta 6 versiones de EnSenias, hasta la adaptación total a sus posibilidades y necesidades de todos.

De las aplicaciones arriba revisadas de otros contextos se desconoce su proceso de validación por parte de los usuarios. En esta ocasión, se pretendió conocer cómo los usuarios potenciales acogen esta iniciativa EnSenias, a modo de validación inicial, cuya continuación natural en cuanto a investigación será proponer estudiar el uso y la efectividad de esta aplicación para los colectivos indicados, mediante un estudio cuasiexperimental que evalúe los beneficios de la misma, una vez conocida su buena acogida. En efecto, según los resultados anteriores, los usuarios declaran la pertinencia y la necesidad de EnSenias, lo que conformaba la primera metacategoría de "Valoración de la plataforma" (VGP), sin ningún lugar a dudas. Y ello se debe a su apuesta firme por la "Usabilidad para aprender la LSP" (palabras) (UPA) y, además, por su superioridad y conveniencia como recurso para "enseñar la LSP" (vocabulario) (UPE) respecto del tradicional libro de LSP, como han puesto de relieve sus propios usuarios. Se procuró reforzar el vínculo positivo que ofrece EnSenias con la "Usabilidad para aprender la lectoescritura" (UPL), al presentar las palabras, su categoría gramatical y la descripción textual, pero ese matiz no ha sido corroborado por los usuarios.

Otras iniciativas tecnológicas se han orientado hacia el perfeccionamiento y la ampliación del señabulario de LS. Así, han aparecido diccionarios digitales, en distintos formatos (DVD, Internet), como el "Diccionario de lengua de signos española" (DILSE), creado por la Confederación Nacional de Sordos Españoles (2000). La plataforma EnSenias también tiene como propósito constituirse en un diccionario propio para la LSP, y así ha sido verificado por los participantes de este estudio, mediante la categoría "Usabilidad de la plataforma como diccionario de palabras” en LS (UPD).

No obstante, lo que más prolifera, dado el problema más acuciante de la población, que es la comunicación y, por tanto, la mayor demanda social de las comunidades sorda y oyente, son los programas online que traducen la LS a comunicación oral, y viceversa. Se trata de recursos tecnológicos muy sofisticados, que están emergiendo en la actualidad desde el terreno de la ingeniería de la programación, que emplean diversos hardware adicionales de captura y reproducción de movimientos (Ibrahim et al., 2017; López-Ludeña et al., 2013), incluso mediante un "avatar" configurable (HETAH, 2012) y sofisticados sistemas, como brazos robóticos (Rajaganapathy et al., 2015). Aun con menor grado de sofisticación, la aplicación EnSenias ha logrado cierta aprobación entre los participantes del estudio para esta funcionalidad, a nivel elemental, como era su propósito.

Algunos de los programas anteriores se presentan como prototipos. En la misma línea se halla EnSenias, que ha pasado por un proceso de validación inicial de sus participantes, antes de ser empleado como recurso para aprender, enseñar, usar y mejorar la LS. Las críticas recibidas de ellos, contempladas mediante la metacategoría "Mejoras [de la plataforma]", han sido escasas y, en su mayoría, han sido consideradas para las versiones optimizadas de la plataforma, hasta la actual versión 6. Las insalvables, denominadas "Limitaciones de la plataforma (LIP)", han sido testimoniales. No obstante, se requiere continuar el proceso de investigación, mudando el diseño de IDB por uno cuasiexperimental, en el cual un grupo de usuarios diferentes actúe de control, usando el libro de LSP y la instrucción habitual en LSP, y otro experimental, que utilicen la aplicación y se beneficien de un curso MOoc (Massive Online Open Courses) innovador para su aprendizaje, dominio y empleo de LSP, contrastando los resultados de unos y otros, mediante evaluación inicial (pretest) y final (postest) tras el tratamiento (uso de EnSenias). 
En cuanto al desarrollo del producto, debe servir de aliento para ir incrementando el vocabulario de la LSP, estimulado desde la publicación del libro de LSP en 1990. Y debe extender su campo de acción para integrar todas las LS de ámbitos afines y cercanos donde cualquier usuario sordo puede trasladarse, tomando como referencia la aplicación Spread Sign (2.0.2), del contexto europeo, que contiene signos del conjunto de países miembros de la Unión Europea (European Sign Language Centre, 2018).

Por último, el desarrollo de la plataforma EnSenias debe contagiar a otros investigadores para que integren, desde el inicio de la programación, el matiz educativo, la construcción-validación de productos con sus usuarios también desde el inicio, y partir de los criterios de accesibilidad y usabilidad universales, para conseguir productos para todos (que integren) y no para unos sectores o comunidades (que segreguen).

\section{Referencias}

Alaín, L. (2019). Desarrollo y validación de una aplicación Web y CD educativa inclusiva para el aprendizaje de la lengua de signos panameña [Tesis de doctorado, Universidad de Granada]. http://hdl.handle. net/10481/56430

Alaín, L., y Vejarano, R. (2016). Alternativas tecnológicas para mejorar la comunicación de personas con discapacidad auditiva en la educación superior panameña. REUGRA, 23, 219-235. http://reugra.es/index.php/ reugra/article/view/62

Anderson, G. L., y Jones, F. (2000). Knowledge generation in educational administration from the inside-out: The promise and perils of site-based, administrator research. Educational Administration Quarterly, 36(3), 428-464. https://doi. org/10.1177/00131610021969056

Bardin, L. (1996). Análisis de contenido. Akal.

Benito, B. de, y Salinas, J. M. (2016). La investigación basada en diseño en tecnología educativa. RIITE. Revista Interuniversitaria de Investigación en Tecnologia Educativa, (0), 44-59. https://revistas.um.es/ riite/article/download/260631/195691/

Cabero-Almenara, J., y Ruiz-Palmero, P. J. (2017). Las Tecnologías de la Información y Comunicación para la inclusión: reformulando la brecha digital.
International Journal of Educational Research and Innovation, (9), 16-30. https://idus.us.es/xmlui/ bitstream/handle/11441/66918/2665-8692-1-PB. pdf ?sequence $=1$ \&isAllowed $=y$

Carr, W., y Kemmis, S. (1986). Teoría critica de la enseñanza. La investigación-acción en la formación del profesorado. Martínez-Roca.

Chacón, E., Aguilar, D., y Sáenz, F. (2014). Desarrollo de una interfaz para el reconocimiento automático del en lengua de señas. MASKAY, 4(1), 14-21. http://dx.doi. org/10.24133/maskay.v4i1.135

Coleman, H., y Unrau, Y. (2005). Analyzing qualitative data. En R. M. Grinnell e Y. Unrau (Eds.), Social work: Research and evaluation. Quantitative and qualitative approaches (pp. 52-76). Oxford University Press.

Confederación Nacional de Sordos Españoles. (2000). DILSE. Diccionario de lengua de signos española [software]. http://www.fundacioncnse.org/tesorolse/ diccionario.html

Costa, M., Leal, G., Diniz, C., Dias, L., y Borcard, L. (2017). Inclusãosocialde deficientes auditivospormeio de Tecnologias assistivas. Anaisdo Encontro Virtualde Documentação em Software Livre e Congresso Internacional de Linguageme Tecnologia Online, 6(1), 1-6. http://www.periodicos.letras.ufmg.br/index.php/anais_linguagem_tecnologia/ article/view/12091/10291

Ensenias V6 (s. f.). Aplicación web EnSenias. http://www. linyadoo.com/ensV6/pages/index.php

European Sign Language Centre (2018). Spread Signs (2.0.2) [aplicación móvil]. Google Play Store. https://play. google.com/store/apps/details?id=com.spreadthesign.androidapp_paid\&hl=es

Garza, R., y Monsivais, J. (2015). Dilo en señas (2.0) [aplicación móvil]. Google Play Store. https://play.google.com/ store/apps/details?id=com.jaguarlabs.lsm\&hl=es

González, G., y Sola, R. (2006). La enseñanza de los sistemas alternativos bajo el prisma de las nuevas tecnologías. Edutec, (15). https://doi.org/10.21556/ edutec.2002.15.547

Grinnell, R. (1997). Social work research \& evaluation: Quantitative and qualitative approaches. Peacock Publishers.

Hernández, C., Pulido, J. L., y Arias, J. E. (2015). Las tecnologías de la información en el aprendizaje de la lengua de señas. Revista de Salud Pública, 17(1), 6173. https://dx.doi.org/10.15446/rsap.v17n1.36935

Hernández, E., Arroyave, A., y Pérez, M. (2016). Recurso educativo web para la enseñanza de lengua de signos colombiana. Ponencia presentada en viII International 
Conference of Adaptive and Accessible Virtual Learning Environment, Fundaciòn Universitaria Tecnológico Comfenalco, Cartagena.

Hernández, R., Fernández, C., y Baptista, P. (2010). Metodología de la investigación. McGraw Hill.

Hernández, R., Rodríguez-Fuentes, A., y Roselli, N. (2019). Editorial. Integración de las TIC a la educación: una mirada desde el aula universitaria. Hamut'ay, 6(3), 9-11. http://dx.doi.org/10.21503/hamu.v6i3.1839

HeTAh (Herramientas Tecnológicas para Ayuda Humanitaria) (2012). Traductor a lengua de señas [software]. http://hetah.net/es

Hidalgo, Y. (2011). Mis manos también hablan. Ministerio de Educación de Panamá. http://www. educapanama.edu.pa/?q=articulos-educativos/ mis-manos-tambien-hablan

Ibrahim, N., Selim, M., y Zayed, H. (2017). An automatic Arabic Sign Language Recognition System (ArSLRS). Journal of King Saud University - Computer and Information Sciences, 30(4), 470-477. https:// doi.org/10.1016/j.jksuci.2017.09.007

Lewin, K. (1946). Action research and minority problems. Journal of Social Issues, 2(4), 34-46. https://doi. org/10.1111/j.1540-4560.1946.tb02295.x

López-Ludeña, V., Barra-Chicote, R., Lutfi, S., Montero, J., y San-Segundo, R. (2013). LSESpeak: A spoken language generator for Deaf people. Expert Systems with Applications, 40(4), 1283-1295. http://dx.doi. org/10.1016/j.eswa.2012.08.062

Miranda, G. M. (2018). Señas nicas (1.0.1) [aplicación móvil]. Google PlayStore.https://play.google.com/store/apps/ details?id=com.marcosmiranda.seniasnicas\&hl=es

Morales, M. R. (2018). Aprende señas: lengua de señas mexicana [aplicación móvil]. Google Play Store. https:// play.google.com/store/apps/details?id=rodolfo. com.systemsolutionand development. aprendiendolsm\&hl=es_CO

Pimentel, D., Walker, R. y Fajardo, M. (1990). Lengua de señas panameñas. SENADIS. http://www.senadis.gob. $\mathrm{pa} / \mathrm{pdf} /$ LENGUA_DE_SENAS-web.pdf
Pressman, R. (2002). Ingeniería del software. McGraw-Hill.

Rajaganapathy, S., Aravind, B., Keerthana, B., y Sivagami, M. (2015). Conversation of sign language to speech with human gestures. Procedia Computer Science, 50, 10-15. https://doi.org/10.1016/j.procs.2015.04.004

Ramírez, F. (2015, abril 6). Entrevistas grupales: los focus group vs. los grupos de discusión. Manual del Investigador. http://manualdelinvestigador.blogspot.com/2015/04/ entrevistas-grupales-los-focus-group-vs.html

Rodríguez, A. (2018). Editorial. Expansión postmoderna tecnológica, escuela inclusiva tecnológica. Revista RETOS XXI, 2(1), 5-12. https://doi.org/10.33412/ retoxxi.v2.1.2055

Rodríguez, A., y Caurcel, M. J. (2019). Aproximación cualitativa del escudriño en psicología educativa. Propósitos y Representaciones, 7(1), 1-9. http:// dx.doi.org/10.20511/pyr2019.v7n1.301

Rodríguez, A., y García, A. (2012). Medios de comunicación y discapacidad. Entre la accesibilidad y la interactividad. Revista ICONO14, 8(1), 303-319. https://doi.org/10.7195/ri14.v8i1.296

Sampieri, R., Collado, C., y Lucio, B. (2010). Sesiones en profundidad o grupos de enfoque. McGraw-Hill.

Secretaría Nacional de Discapacidad de Panamá (sEnAdis) (2018). Lengua de señas panameñas. http://www.senadis.gob.pa/pdf/LENGUA_DE_SENAS-web.pdf

Stenhouse, L. (2007). Investigación y desarrollo del curriculo. Morata.

Universidad de Granada. Vicerrectorado de Investigación y Transferencia. (s. f.). Solicitar evaluación y certificado de un comité de ética. Solicitud investigación humana. https://investigacion.ugr.es/apoyo/ comite-etica/evaluacion.

Universidad Nacional de Costa Rica (2012). Sistema de la Lengua de Señas Costarricense (LESCO). http:// www.cenarec-lesco.org/index.php/61-mosaico/55lengua-de-senas-costarricense-lesco

Cómo citar este artículo: Rodríguez-Fuentes, A., Alain, L., y García García, F. (2020). EnSenias: herramienta tecnológica para aprender, enseñar, mejorar y usar la lengua de signos panameña. Íkala, Revista de Lenguaje y Cultura, 25(3), 663-678. https:/doi.org/10.17533/udea.ikala.v25n03a05 